\title{
Characterizations of Ischemic Stroke Complications in Cardiac Myxoma Patients at a Single Institution in Eastern China
}

This article was published in the following Dove Press journal: Neuropsychiatric Disease and Treatment

\author{
Yanlei Zhang' \\ Zusen $\mathrm{Ye}^{\mathrm{I}}$ \\ Yun $\mathrm{Fu}^{2}$ \\ Zheng Zhang' \\ Qiang $\mathrm{Ye}^{\prime}$ \\ Feichi Chen (DD' \\ Jianhua Cheng'
}

'Department of Neurology, The First Affiliated Hospital of Wenzhou Medical University, Wenzhou, Zhejiang Province 325000, People's Republic of China; ${ }^{2}$ Department of Cardiac Surgery, The First Affiliated Hospital of Wenzhou Medical University, Wenzhou, Zhejiang Province 325000, People's Republic of China
Correspondence: Jianhua Cheng Department of Neurology, The First Affiliated Hospital of Wenzhou Medical University, Nanbaixiang, Ouhai District, Wenzhou 325000, Zhejiang Province, People's Republic of China

Tel +86 57755579365

Fax +86 57755578999

Email chengjianhua@wmu.edu.cn
Background: Cardiac myxoma is the most common primary cardiac tumor. Even though it rarely causes a stroke, it is an important risk factor. Here, we compared our clinical experience in managing myxoma patients who developed stroke complications with those who did not present with this condition at the First Affiliated Hospital of Wenzhou Medical University.

Patients and Methods: The medical records were reviewed of 160 cardiac myxoma patients who were treated in our facility from January 2006 to December 2019. They were separated into either a stroke group or non-stroke group.

Results: Cardiac obstructive symptoms, embolic events and constitutional symptoms were observed in 92 (57.7\%), 25 (15.6\%) and 18 (11.2\%) patients, respectively. Among 23 cardiac myxoma ischemic stroke patients, hypoesthesia (60.9\%), hemiparesis $(56.5 \%)$ and facial paresis $(47.8 \%)$ were the three most common neurological symptoms. The middle cerebral artery $(82.6 \%)$ was the most commonly affected vessel, whereas $73.9 \%$ of the ischemic patients had multiple stroke lesions. Binary logistic regression analysis showed that coronary heart disease and tumor sizes were independently associated in the stroke group $(p<0.05)$. The 10 years cumulative survival rate was $87.9 \%$ for all patients after surgical intervention. There was no significant difference in the 10 years cumulative survival rate between the two groups $(80.0 \%$ vs $88.9 \%, \mathrm{p}=0.274>0.05)$.

Conclusion: The three most common neurological symptoms (hypoesthesia, hemiparesis and facial paresis), the middle cerebral artery and multiple lesions involvements were the definitive markers of patients afflicted with cardiac myxoma stroke. Small tumor sizes were independently associated with these patients. Surgical resection is a relatively safe procedure for treating both the stroke and non-stroke patients.

Keywords: cardiac myxoma, embolic complication, ischemic stroke, surgery

\section{Introduction}

At autopsy, the incidence of primary tumors of the heart is very rare. Their incidence ranges only between $0.001 \%$ and $0.3 \%{ }^{1}$ In these rare instances, cardiac myxomas are the most common pathology in $80 \%$ of the cases. ${ }^{2}$ Cardiac myxomas occur most frequently in 30 to 40 years old patients and they are twice as prevalent in females as in males. ${ }^{3} 75 \%$ of the cardiac myxomas originate from the left atrium whereas they are less commonly found in the right atrium, bilateral atria, heart valves, and only $3 \%-4 \%$ are detected in either ventricle. ${ }^{2}$

Systemic embolization of cardiac myxoma occurs in up to $45 \%$ of the patients, half of which develop in the central nervous system. ${ }^{4}$ The cerebrovascular region is 
the most common site of myxoma emboli. These strokes were reported to range from $21 \%$ to $29 \%$. $^{3,5,6}$ The most common neurologic symptoms were hemiparesis (49\%), aphasia $(27.9 \%)$, and alteration of consciousness $(23.1 \%){ }^{7}$

The current study is based on our clinical experience with cardiac myxomas over a period of 14 years at the First Affiliated Hospital of Wenzhou Medical University. We focus here on describing the symptoms of cardiac myxomas and ischemic stroke complications that develop in these patients. In addition, it deals with stroke lesion patterns, and analysis of the stroke risk factors. Long-term survival was also evaluated through follow-ups on patients that underwent cardiac myxoma extirpation.

\section{Methods}

\section{Study Population}

The medical records of 160 consecutive patients with pathologically confirmed cardiac myxoma were reviewed at the First Affiliated Hospital of Wenzhou Medical University between January 2006 and Dec 2019. The items in their electoral clinical records that were reviewed include: medical history, operative reports, neurologic examination, pathological results, computed tomography (CT) or magnetic resonance imaging (MRI) of the brain, echocardiography and ECG. Ischemic stroke patients were diagnosed based on either CT or MRI results.

\section{Clinical Variables}

Demographic data, stroke risk factors (ie smoking and drinking habits, history of hypertension, diabetes mellitus, hyperlipidaemia, and coronary heart disease), clinical presentations of myxoma and the neurologic examination results obtained upon hospital admission were recorded. Echocardiography analysis evaluated heart valvular structure, ejection fraction, left atrial diameter along with occurrence of cardiac myxomas. The maximum tumor diameter at the time of removal was also recorded.

Locations of cerebral infarcts and the affected cerebral artery were identified based on the results of either CT or MRI. Neuroimaging determined the infarct location based on evaluation of the vascular distribution in the anterior and/or posterior circulation.

\section{Clinical Follow-Up}

After hospital discharge, outcome was monitored for periods lasting from 0.5 to 14.2 years. Our evaluation procedure entailed either clinical visits, or telephone interviews to record patients, survival.

\section{Statistical Analysis}

Continuous variables were expressed as mean \pm SD (standard deviation) or median (IQR, interquartile range), depending on the normality of data distribution and categorical variables were expressed as a percentage. Categorical variables were analyzed using the $\chi^{2}$ test, continuous variables were analyzed using the Student's $t$-test, and non-normally distributed continuous variables were analyzed using the Mann-Whitney rank sum tests. A binary logistic regression analysis was used to assess variables associated with determining myxoma-related ischemic stroke. Kaplan-Meier methods estimated survival rate after surgical resection of cardiac myxoma.

\section{Results}

The mean age of the 160 patients diagnosed with cardiac myxoma was $56.58 \pm 12.89$ years (range 14-80). There were 107 (66.9\%) female patients, and the ratio of female: male was 2.02:1. There were 142 left atrial myxomas $(88.7 \%), 13$ right atrial myxomas $(8.1 \%), 2$ left ventricle myxomas (1.2\%), 2 right ventricle myxomas (1.2\%), and 1 right atrial and ventricle myxoma $(0.6 \%)$.

Cardiac obstructive symptoms were the most common findings and they were present in 92 patients (48.1\%). Among them, chest pain or discomfort was the most common (54.2\%), dyspnea and palpitations were evident in 43 and 13 patients, respectively. Embolic events occurred in 25 patients $(22.0 \%)$. Embolic events were the initial manifestations in these 25 patients diagnosed with cardiac myxoma. 23 out of 25 patients with an embolism had brain infarct symptoms. Among these 23 ischemic stroke patients, one patient had a left limb embolism and stroke at the same time, and another patient had both an acute ischemic stroke and acute myocardial infarction. Another two in a group of 25 embolic patients had the following characteristics: one 25 years old patient without any predisposing risk factors presented with acute myocardial infarction, whereas another had aorta emboli. 18 patients $(16.9 \%)$ had constitutional symptoms, including fever, fatigue, and weight loss. Fatigue occurred in 6 patients and it was the most frequent constitutional symptom. Weight loss and fever were observed in 4 and 3 different cases, respectively. 60 patients $(37.5 \%)$ were asymptomatic (Table 1$)$. In these cases, the myxoma was found by accident during a routine 
Table I Clinical Presentations of 160 Patients with Cardiac Myxoma

\begin{tabular}{|l|l|}
\hline Symptom & $\mathbf{n}(\%)$ \\
\hline Cardiac obstructive symptoms & $92(57.5)$ \\
Chest pain or discomfort & $68(42.5)$ \\
Dyspnea & $43(26.9)$ \\
Palpitation & $13(8.1)$ \\
Cough & $12(7.5)$ \\
Syncope & $7(4.3)$ \\
\hline Embolism & $25(15.6)$ \\
Brain & $23(14.3)$ \\
Limb & $1(0.6)$ \\
Acute myocardial infarction & $2(1.2)$ \\
Aortic saddle embolism & $1(0.6)$ \\
\hline Constitutional symptoms & $18(11.3)$ \\
Fatigue & $15(9.4)$ \\
Weight loss & $5(3.1)$ \\
Fever & $2(1.2)$ \\
Incidental & $60(37.5)$ \\
\hline
\end{tabular}

echocardiography exam performed as part of a physical examination and for other reasons.

Among these 23 ischemic stroke patients, hypoesthesia $(60.9 \%)$, hemiparesis $(56.5 \%)$ and facial paresis $(47.8 \%)$ were three of the most common neurological symptoms (Table 2). The three most common underlying infarct regions were the front lobe $(39.1 \%)$, the parietal lobe $(39.1 \%)$, and the basal ganglion (34.7\%) (Table 3). The most common affected vessel was the middle cerebral artery $(82.6 \%)$. The anterior circulation was involved in 15 patients, whereas the posterior circulation was involved in 4 others. Both the

Table 2 Neurological Symptoms in 23 Stroke Patients

\begin{tabular}{|l|l|}
\hline Neurological Symptom & n (\%) \\
\hline Hypoesthesia & $14(60.9 \%)$ \\
Hemiparesis & $13(56.5 \%)$ \\
Facial paresis & $\mathrm{I}(\mathbf{( 4 7 . 8 \% )}$ \\
Conscious alteration & $7(30.4 \%)$ \\
Hemiplegia & $7(30.4 \%)$ \\
Dysarthria & $7(30.4 \%)$ \\
Aphasia & $6(26.1 \%)$ \\
Dizziness & $6(26.1 \%)$ \\
Ataxia & $2(8.7 \%)$ \\
Vision disturbance & $2(8.7 \%)$ \\
Diplopia & $2(8.7 \%)$ \\
Seizures & $1(4.3 \%)$ \\
Gaze & $\mathrm{I}(4.3 \%)$ \\
Headache & $\mathrm{I}(4.3 \%)$ \\
\hline
\end{tabular}

Table 3 Locations of Cerebral Infarcts and Affected Cerebral Artery in 23 Stroke Patients

\begin{tabular}{|l|l|}
\hline Location & $\mathbf{n}(\%)$ \\
\hline Location of infarct & $9(39.1)$ \\
\hline Frontal & $9(39.1)$ \\
Parietal & $8(34.7)$ \\
Basal ganglion & $6(26.1)$ \\
Corona radiate & $5(21.7)$ \\
Temporal & $5(21.7)$ \\
Occipital & $4(17.3)$ \\
Cerebellum & $3(13.0)$ \\
Brainstem & $2(8.7)$ \\
Insula & $2(8.7)$ \\
Thalamic & \\
\hline Affected cerebral artery & $15(65.2)$ \\
\hline Anterior circulation & $4(17.4)$ \\
Both side MCA & $11(47.8)$ \\
Single side MCA & $4(17.4)$ \\
Anterior and posterior circulation & $2(8.7)$ \\
Both side MCA+PCA & $1(4.3)$ \\
Single side MCA+PCA & $1(4.3)$ \\
Single side MCA+BA & $4(17.4)$ \\
Posterior circulation & $2(8.7)$ \\
PCA & $2(8.7)$ \\
BA & \\
\hline
\end{tabular}

Abbreviations: MCA, middle cerebral artery; PCA, posterior cerebral artery; BA, basal artery.

anterior and posterior regions were involved in 4 patients (Table 3). 17 (73.9\%) ischemic patients had multiple stroke lesions. But there were no differences between single lesion and multiple lesions in the anterior circulation and posterior circulation (Table 4). 3 patients received thrombolytic therapy with recombinant tissue plasminogen activator (rTPA), whereas neither of them had an intracranial hemorrhage. After ischemic stroke, 21 patients received antiplatelet, whereas two others accepted anticoagulation therapy. These

Table 4 Comparisons of Site and Number of Infarcts

\begin{tabular}{|l|l|l|l|}
\hline Infarct Side & $\begin{array}{l}\text { Single } \\
(\mathbf{n = 6})\end{array}$ & $\begin{array}{l}\text { Multiple } \\
(\mathbf{n}=\mathbf{1 7})\end{array}$ & $\boldsymbol{P}$ \\
\hline Anterior circulation & 4 & 12 & 1.000 \\
$\quad$ Left & 3 & 3 & 0.279 \\
$\quad$ Right & $\mathrm{I}$ & 5 & 1.000 \\
$\quad$ Bilateral & - & 4 & - \\
Anterior and posterior & - & 3 & - \\
circulation & & & 0.270 \\
Posterior circulation & 2 & 2 & \\
\hline
\end{tabular}


Table 5 Comparison of Factors Between the Stroke and Non-Stroke Groups

\begin{tabular}{|l|l|l|l|l|}
\hline & Cardiac Myxoma (n=160) & Stroke (n=23) & No-Stroke (n=137) & $P$ \\
\hline Age, years & $56.58 \pm 12.89$ & $60.48 \pm 9.69$ & $55.93 \pm 13.27$ & 0.118 \\
Female (\%) & $107(66.9)$ & $13(56.5)$ & $94(68.6)$ & 0.254 \\
Hypertension (\%) & $47(29.4)$ & $10(43.5)$ & $37(27.0)$ & 0.109 \\
Diabetes mellitus (\%) & $23(14.4)$ & $3(13.0)$ & $20(14.6)$ & 0.844 \\
Hyperlipidemia (\%) & $14(8.8)$ & $6(26.1)$ & $7(5.8)$ & 0.001 \\
Coronary heart disease (\%) & $12(7.5)$ & $5(21.7)$ & $10(7.3)$ & 0.005 \\
Atrial fibrillation (\%) & $12(7.5)$ & $2(8.7)$ & $12(8.8)$ & $0.84 \mid$ \\
Current smoking (\%) & $15(9.4)$ & $3(13.0)$ & $5(3.6)$ & $30(21.9)$ \\
Current drinking (\%) & $5(3.1)$ & 0 & $64.05 \pm 7.74$ \\
Valvular heart disease (\%) & $34(23.1)$ & $4(17.4)$ & $39.21 \pm 6.86$ \\
Ejection fraction, \% & $64.16 \pm 7.39$ & $64.77 \pm 4.98$ & $39.61 \pm 6.40$ & 1.000 \\
Left atrial diameter before operation, mm & $39.27 \pm 6.78$ & $3.43 \pm 1.05$ & 0.625 \\
Tumor size, cm & $4.41 \pm 1.67$ & $21(91.3)$ & $121(88.3)$ \\
Tumor in left atrium (\%) & $142(88.6)$ & & 0.670 \\
\hline
\end{tabular}

23 patients had surgical resection performed 3-4 weeks after stroke onset. There was no intracerebral hemorrhagic conversion of the ischemic infarcts and no recurrent ischemic strokes before an operation.

Basic characteristics, stroke risk factors, tumor sizes, and echocardiography findings (ie ejection fraction, and left atrium diameter) were compared between the stroke and non-stroke group. The tumors were significantly smaller in the stroke group, and hyperlipidemia and coronary heart disease were significantly more frequent in the stroke group. There were no significant differences among the other stroke risk factors, the ejection fraction, and left atrial diameter (Table 5). Binary logistic regression showed that tumor size and coronary heart disease were independently associated with myxoma-related stroke ( $p=$ 0.003; OR 0.592; 95\% CI (0.419-0.837), $p=0.009$; OR 5.666; 95\% CI (1.542,20.821), respectively) after adjusted above mentioned factors (Table 6). Taken together, the results suggest that the risk of stroke is higher in patients having small tumors.

There was no perioperative mortality in these 160 cases. Only $12(7.5 \%)$ of the 160 patients had complications related to surgery. Two patients had a postoperative ischemic stroke and they required rehabilitation for several months. Other complications included pulmonary infection (2 patients), pleural effusion (2 patients), pleural effusion and pulmonary infection ( 2 patients), deep vein thrombosis (DVT) (2 patients), acute myocardial infarction (1 patient), and acute pancreatitis ( 1 patient). All of the ischemic stroke patients underwent surgical resection of a cardiac myxoma 3 to 4 weeks after a stroke. No intracerebral hemorrhage or recurrence of an ischemic stroke appeared during the perioperative period. All of these complications completely resolved except in stroke patients during their hospitalization.

Among these 160 patients, 132 patients were available for follow-up after undergoing a cardiac myxoma operation, while $28(17.5 \%)$ patients failed to participate (3 were in the stroke group, and 25 were in the non-stroke group). In this study, the results of these 28 patients who failed to participate were excluded from the study. The follow-up period lasted from 0.42 to 14.17 years (median 4.46 years, interquartile range 1.77-7.34). During the follow-up period, 1 patient in the stroke group had another stroke, and atrial myxoma recurred in two patients (2/ $132,1.5 \%$ ). 5 patients died during a follow-up (one in the stroke group, and four in the non-stroke group), but their mortality was not different between the two groups $(p>$ $0.05)$. The 10 years cumulative survival rate was $87.9 \%$

Table 6 Binary Logistic Regression Model for Determining Myxoma-Related Ischemic Stroke

\begin{tabular}{|l|l|l|}
\hline Variables & Adjusted OR (95\% Cls) & $\boldsymbol{p}$ \\
\hline Age & $1.033(0.963,1.082)$ & 0.179 \\
Female & $1.168(0.345,3.948)$ & 0.803 \\
Hypertension & $1.608(0.557,4.642)$ & 0.380 \\
Diabetes mellitus & $0.357(0.078,1.644)$ & 0.186 \\
Hyperlipidemia & $3.092(0.832,11.495)$ & 0.092 \\
Atrial fibrillation & $0.504(0.060,4.205)$ & 0.526 \\
Coronary heart disease & $5.666(1.542,20.821)$ & 0.009 \\
Valvular heart disease & $0.720(0.148,3.514)$ & 0.685 \\
Tumor size & $0.592(0.419,0.837)$ & 0.003 \\
\hline
\end{tabular}




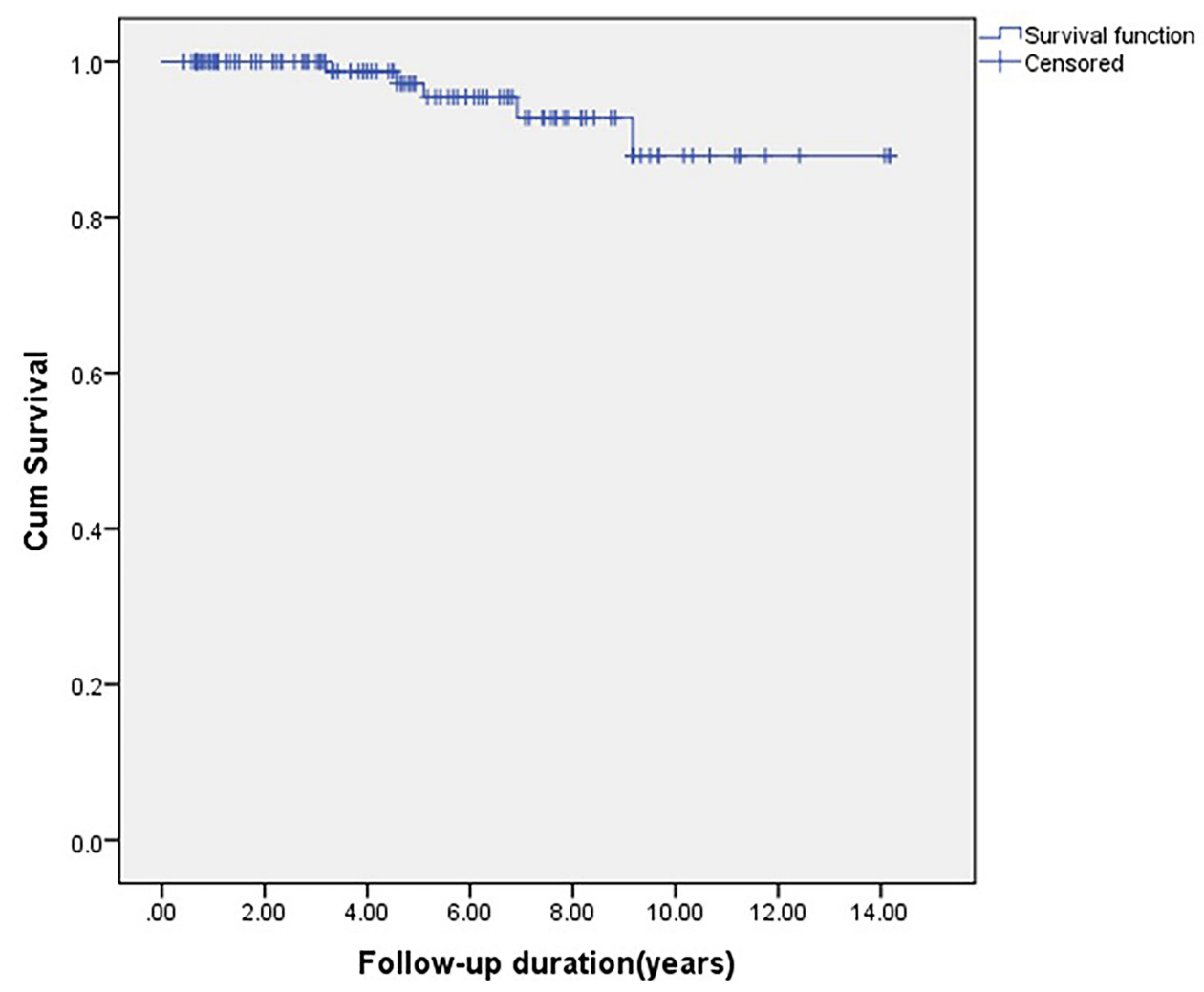

Figure I Kaplan-Meier plot for cumulative proportion of survival after surgery for cardiac myxoma in studied population. Cum survival = cumulative survival.

for all patients after an operation (Figure 1). In the stroke group, the 10 years cumulative survival rate was $80.0 \%$ after an operation. In the non-stroke group, the 10 years cumulative survival rate was $88.9 \%$ after operation, but this difference between the two groups was not significant $(p=0.274>0.05)$ (Figure 2).

\section{Discussion}

In our study, stroke occurred in $14.3 \%$ of the patients whereas limb embolism was seen in only $0.6 \%$ of those individuals. And, acute myocardial infarction occurred in $1.2 \%$ of the cases. Embolic events may involve the central nervous system, the extremity, visceral, coronary, and pulmonary emboli. ${ }^{8-11}$ Our results also showed the involvement of ischemic stroke, the extremity, coronary, and aorta emboli. Embolic events in atrial myxoma are thought to be secondary to embolization of tumor particles. Emboli can also be composed of thrombotic material covered with tumor cells. ${ }^{12,13}$ Myxomas with an irregular tumor surface, tumor location, are more frequently associated with production of emboli. ${ }^{13,14}$

There is no consensus regarding whether or not tumor size is related to the likelihood of a stroke. Some previous reports showed that the mobility and morphology of the tumor, but not its size, appears to be related to its embolic risk. ${ }^{5,15,16}$ On the other hand, some studies showed that smaller tumors with an irregular surface were more common in embolic events. ${ }^{6,17}$ In a previous report from China, small tumors with a diameter of less than $3 \mathrm{~cm}$ were more common in patients with cerebral infarction, indicating that small tumors had a higher risk of stroke. The causes might be related to the early occurrence of tissue detachment and thrombosis in these patients. Therefore, the effect of complications should not be ignored because of small size tumor. Once a tumor is found, early surgery is warranted to reduce the risk of recurrent stroke. ${ }^{6}$ Our study shows that the tumor sizes of the atrial myxoma in the stroke group were shorter than in the no-stroke group $(3.43 \pm 1.05 \mathrm{~cm}$ vs $4.54 \pm 1.70 \mathrm{~cm}$, $\mathrm{p}=0.003$ ). Nevertheless, binary logistic regression showed that tumor sizes were independently associated with myxoma-related stroke.

Single cerebral vessel (mostly the middle cerebral artery), multiple regional involvements and solitary left atrial myxoma are very definitive characteristics in patients with cardiac myxoma stroke. The most commonly 


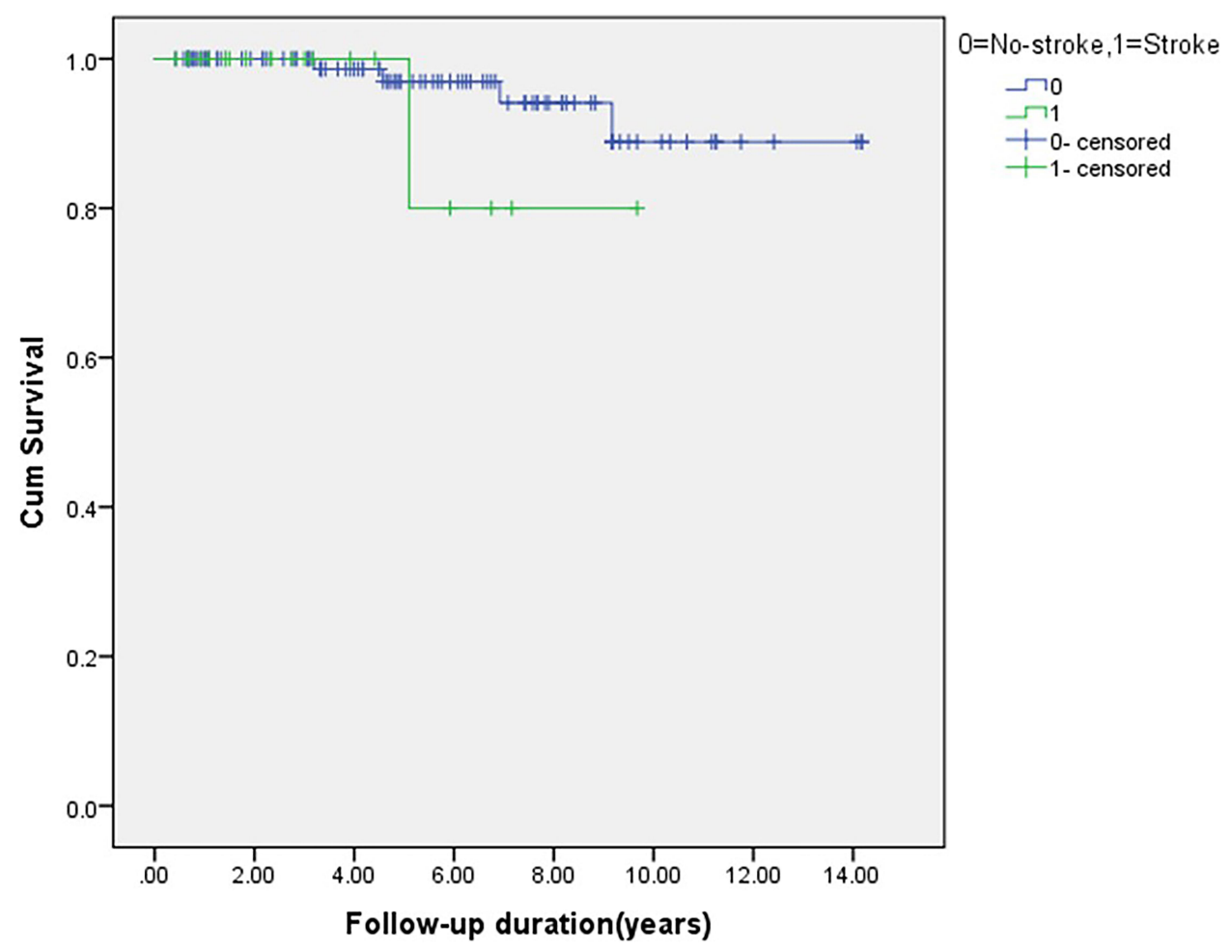

Figure 2 Kaplan-Meier curves for during follow-up in cardiac myxoma patients after surgical resection. There was no significant difference in survival rate between the stroke group and no-stroke group $(p=0.197)$. Cum survival $=$ cumulative survival.

affected cerebral vessel was the middle cerebral artery and affected areas were the basal ganglion, cerebellum, parietal, and temporal regions. ${ }^{7}$ The predominant patterns in the lesion topography of cardiac myxoma in ischemic stroke patients were multiple lesions and middle cerebral artery territory involvement. Neuroimaging showed bilateral, supratentorial, and infratentorial embolic infarcts. ${ }^{18}$ It was reported that the main lesion pattern was multiple lesions $(72.7 \%)$, followed by middle cerebral artery regional lesions $(63.6 \%) .^{5}$ Our findings are similar because $73.9 \%$ of the ischemic patients had multiple stroke lesions and the middle cerebral artery $(82.6 \%)$ was the most common affected vessel.

Among these 23 ischemic stroke patients in this report, 3 patients received thrombolytic therapy with recombinant tissue plasminogen activator (rTPA), and none of these individuals experienced an intracranial hemorrhage. Although there are no clear guidelines and there is lack of consensus regarding the type of treatment that is needed for managing acute stroke secondary to cardiac myxoma embolization, there are about 20 cases of thrombolysis with rTPA. The therapeutic effects of rTPA were variable. Most patients experienced an improvement, whereas some symptoms remained unchanged. On the other hand, some patients even had an intracranial hemorrhage. ${ }^{19}$ The efficacy of rTPA may be reduced in patients whose embolus is mainly composed of tumor tissue rather than a thrombus. ${ }^{20}$ Therefore, mechanical thrombectomy may be a better option than thrombolysis for treatment of patients who have an occluded large artery. ${ }^{19,20}$

The 10 years cumulative survival rate was more than $85 \%$ in cardiac myxoma patients who underwent surgery. This finding agrees with another report involving about 64 cardiac myxoma patients who were operated on. Their 10 years cumulative survival rate was about $84 \%{ }^{21}$ Even though there are some other earlier reports describing the long-time outcome of cardiac myxoma with and without embolization, the current report deals just with the longtime outcome of cardiac myxoma with and without stroke. Both of them 10 years cumulative survival rate were more than $80 \%$. The lack of difference in survival between the two groups shows that surgical resection is a relatively safe procedure for treating cardiac myxoma patients in both the stroke and the non-stroke groups.

This study has some limitations. Firstly, because of its retrospective design in using medical records, the results of this study may be limited. Secondly, selection bias may have occurred because the enrolled patients were drawn only 
from a single-center. Thirdly, in some procedures, myxomas were divided into two types according to the criteria described in previous clinicopathologic studies: type 1, with an irregular surface and a soft consistency, and type 2 , with a regular surface and a compact consistency. ${ }^{12,13}$ However, such descriptions and classifications were not provided in the surgical records of surgeons.

\section{Conclusions}

This report provides clinical characterizations and clinical outcome of ischemic stroke complications in cardiac myxoma patients. The three most common neurological symptoms were hypoesthesia, hemiparesis and facial paresis. These conditions accompanied the middle cerebral artery and multiple regional involvement which were the most apparent characteristics in the cardiac myxoma stroke patients. Small tumor sizes were independently associated with these patients. Surgical resection is a relatively safe and curative option for cardiac myxoma and cardiac myxoma stroke patients.

\section{Ethics Statement}

The study protocol was approved by the Research Ethics Board of the First Affiliated Hospital of Wenzhou Medical University. Due to the retrospective, non-interventional and anonymous nature of this study, informed consent from patients was waived.

\section{Acknowledgments}

This research was supported by Zhejiang Provincial Natural Science Foundation of China under Grant No. LY17H090014. We appreciate very much the excellent support and guidance provided by Peter Reinach in editing our manuscript.

\section{Disclosure}

The authors report no conflicts of interest in this work.

\section{References}

1. Reynen K. Frequency of primary tumors of the heart. Am J Cardiol. 1996;77(1):107. doi:10.1016/S0002-9149(97)89149-7

2. Reynen K. Cardiac myxomas. $N$ Engl $J$ Med. 1995;333 (24):1610-1617. doi:10.1056/NEJM199512143332407

3. Pinede L, Duhaut P, Loire R. Clinical presentation of left atrial cardiac myxoma: a series of 112 consecutive cases. Medicine. 2001;80 (3):159-172. doi:10.1097/00005792-200105000-00002
4. Jain RS, Nagpal K, Jain R, et al. Acute psychosis presenting as a sole manifestation of left atrial myxoma: a new paradigm. Am J Emerg Med. 2014;32(12):1556.e3-5. doi:10.1016/j.ajem.2014.05.006

5. Lee SJ, Kim JH, Na CY, et al. Eleven years' experience with Korean cardiac myxoma patients: focus on embolic complications. Cerebrovasc Dis. 2012;33(5):471-479. doi:10.1159/000335830

6. Cao GF, Bi Q, Cao L, et al. The clinical characteristics of stroke in young patients with cardiac myxoma. Zhonghua Nei Ke Za Zhi. 2017;56(4):263-267. doi:10.3760/cma.j.issn.0578-1426.2017.04.005

7. Yuan SM, Humuruola G. Stroke of a cardiac myxoma origin. Rev Bras Cir Cardiovasc. 2015;30(2):225-234. doi:10.5935/16789741.20150022

8. Ma G, Wang D, He Y, et al. Pulmonary embolism as the initial manifestation of right atrial myxoma: a case report and review of the literature. Medicine (Baltimore). 2019;98(51):e18386. doi:10.1097/MD.0000000000018386

9. Mathew R, Agrawal N, Aggarwal P, et al. Atrial myxoma presenting as acute bilateral limb ischemia. J Emerg Med. 2019;57(5):710-712. doi:10.1016/j.jemermed.2019.06.006

10. McGowan AR, Thibodeau C, McGowan A. Intracranial and visceral arterial embolization of a cardiac myxoma that was treated with endovascular stent-retriever therapy. Interv Neuroradiol. 2016;22 (5):535-539. doi:10.1177/1591019916653939

11. Peters MJ, Tuwairqi KW, Farah MG. A case of infected left atrial myxoma presenting as ST-Elevation Myocardial Infarction (STEMI). Am J Case Rep. 2019;20(17):1930-1935. doi:10.12659/ AJCR.918192

12. Boyacıoğlu K, Kalender M, Dönmez AA, et al. Outcomes following embolization in patients with cardiac myxoma. J Card Surg. 2017;32 (10):621-626. doi:10.1111/jocs. 13220

13. He DK, Zhang YF, Liang Y, et al. Risk factors for embolism in cardiac myxoma: a retrospective analysis. Med Sci Monit. 2015;21:1146-1154. doi:10.12659/MSM.893855

14. Liu Y, Wang J, Guo L, et al. Risk factors of embolism for the cardiac myxoma patients: a systematic review and metanalysis. $B M C$ Cardiovasc Disord. 2020;20(1):348. doi:10.1186/s12872-020-01631-w

15. Wen XY, Chen YM, Yu LL, et al. Neurological manifestations of atrial myxoma: a retrospective analysis. Oncol Lett. 2018;16 (4):4635-4639. doi:10.3892/ol.2018.9218

16. Stefanou MI, Rath D, Stadler V, et al. Cardiac myxoma and cerebrovascular events: a retrospective cohort study. Front Neurol. 2018;9:823. doi:10.3389/fneur.2018.00823

17. Loire R. Myxoma of the left atrium, clinical outcome of 100 operated patients. Arch Mal Coeur Vaiss. 1996;89(9):1119-1125.

18. Liao WH, Ramkalawan D, Liu JL, et al. The imaging features of neurologic complications of left atrial myxomas. Eur $J$ Radiol. 2015;84(5):933-939. doi:10.1016/j.ejrad.2015.02.005

19. Barghouthi T, Giugliano A, Kim-Shapiro JWL, et al. Pearls \& Oysters: IV and mechanical thrombolysis for ischemic stroke secondary to cardiac myxoma. Neurology. 2019;93(22):975-977. doi:10.1212/WNL.0000000000008549

20. Dong M, Ge Y, Li J, et al. Intravenous thrombolysis for pure pontine infarcts caused by cardiac myxoma: a case report and literature review. Int $J$ Neurosci. 2020;130(6):635-641. doi:10.1080/ 00207454.2019.1702537

21. Perek B, Misterski M, Stefaniak S, et al. Early and long-term outcome of surgery for cardiac myxoma: experience of a single cardiac surgical center. Kardiol Pol. 2011;69(6):558-564. 


\section{Publish your work in this journal}

Neuropsychiatric Disease and Treatment is an international, peerreviewed journal of clinical therapeutics and pharmacology focusing on concise rapid reporting of clinical or pre-clinical studies on a range of neuropsychiatric and neurological disorders. This journal is indexed on PubMed Central, the 'PsycINFO' database and CAS, and is the official journal of The International Neuropsychiatric Association (INA). The manuscript management system is completely online and includes a very quick and fair peer-review system, which is all easy to use. Visit http://www.dovepress.com/testimonials.php to read real quotes from published authors.

Submit your manuscript here: https://www.dovepress.com/neuropsychiatric-disease-and-treatment-journal 